\title{
Spinal cord magnetic resonance imaging demonstrates sensory neuronal involvement and clinical severity in neuronopathy associated with Sjögren's syndrome
}

\author{
K Mori, H Koike, K Misu, N Hattori, M Ichimura, G Sobue
}

\begin{abstract}
Objectives-To determine spinal cord MRI findings in neuronopathy associated with Sjögren's syndrome and their correlation with severity of sensory impairment.

Methods-Clinical and electrophysiological features, pathological findings in the sural nerve, and hyperintensity on $\mathrm{T} 2$ * weighted MRI in the spinal dorsal columns were evaluated in 14 patients with neuronopathy associated with Sjögren's syndrome.

Results-Of 14 patients, 12 showed high intensity by $\mathrm{T} 2{ }^{\star}$ weighted MRI in the posterior columns of the cervical cord. High intensity areas were seen in both the fasciculus cuneatus and gracilis in nine patients, who showed severe and widespread sensory deficits in the limbs and trunk; these patients also had a high frequency of autonomic symptoms. Somatosensory evoked potentials often could not be elicited. Hyperintensity restricted to the fasciculus gracilis was seen in three patients, who showed sensory deficits restricted to lower limbs without trunk involvement, or with only partial limb involvement; no autonomic symptoms were noted. The two patients who did not show high intensity areas in the dorsal columns showed restricted sensory involvement in the limbs. All patients showed axonal loss predominantly affecting large fibres, without axonal sprouting. Conclusions-High intensity areas on $\mathrm{T} 2$ * weighted MRI in the spinal dorsal columns reflect the degree of sensory neuronal involvement in neuronopathy associated with Sjögren's syndrome; this finding could also be a helpful marker for estimating severity of this neuronopathy.
\end{abstract}

Neurology, Nagoya University School of Medicine, Nagoya 466-8550 Japan K Mori

H Koike

K Misu

N Hattori

M Ichimura

G Sobue

Correspondence to: Dr Gen Sobue

sobueg@med.nagoya-u.ac.jp

Received 28 February 2001 and in revised form 21 May 2001

Accepted 8 June 2001 peripheral vascular circulatory deficits represent the often seen autonomic manifestations. ${ }^{4}$ By contrast, motor impairment is usually absent or very mild. ${ }^{1-3}$ The distribution and extent of sensory as well as autonomic involvement is remarkably variable among patients. Some patients show only mild and restricted sensory deficits in the distal portions of limbs in the mutiple mononeuropathic type, whereas other patients show profound sensory involvement over the entire body associated with severe sensory ataxia. ${ }^{1-3}$ 5-10

Dorsal root ganglionitis with $\mathrm{T}$ cell infiltration has been reported as the major lesion responsible for this sensory neuronopathy. ${ }^{11}$ Dorsal column involvement on spinal cord MRI, which may reflect degeneration of the posterior columns of the spinal cord secondary to the sensory ganglionopathy, has been demonstrated in anecdotal case reports. ${ }^{12-14}$ However, this MRI finding has not been assessed systematically in neuronopathy associated with Sjögren's syndrome or correlated with the extent and nature of sensory involvement to determine its usefulness as a diagnostic and prognostic tool. We therefore investigated MRI findings in 14 patients with neuronopathy associated with Sjögren's syndrome showing the extent and nature of sensory symptoms, and assessed the importance of MRI for evaluating sensory neuronal involvement.

Patients and methods

Fourteen patients with neuronopathy associated with Sjögren's syndrome who had been referred to the Hospital of Nagoya University School of Medicine and its affiliated hospitals were the subject of this study. These patients consisted of 11 women and three men, with ages ranging from 51 to 75 years (mean (SD) $64.8(6.8)$ ). They had been followed up for 2 to 16 years. All patients fulfilled criteria for primary Sjögren's syndrome and peripheral neuronopathy. The diagnosis of primary Sjögren's syndrome was established by the criteria proposed by the Diagnostic Committee of Health and Welfare of Japan in $1977^{15}$ and by the diagnostic criteria described by the European Community in $1993 .{ }^{16}$ These included symptoms of xerophthalmia, symptoms of xerostomia, objective evidence of keratoconjunctivitis (both an abnormal Schirmer test with less than $5 \mathrm{~mm}$ of wetting of a paper strip at 5 minutes and an abnormal Rose Bengal score exceeding 3 according to the van 
Bijsterveld scoring system); chronic lymphocytic sialoadenitis on a minor salivary gland biopsy specimen (more than one lymphocytic focus $/ 4 \mathrm{~mm}^{2}$ ), abnormal salivary gland scintigraphy or sialography, decreased salivary flow (less than $10 \mathrm{ml} / 10 \mathrm{~min}$ in a gum test), and the presence of either SS-A or SS-B autoantibodies. Other collagen diseases (systemic lupus erythematosus, rheumatoid arthritis, mixed connective tissue disease, progressive systemic sclerosis, polyarteritis nodosa, polymyositis, and Churg-Strauss syndrome) were extensively screened for according to the diagnostic criteria appropriate to each condition, but no such cases were found.

Patients underwent neurological examinations, blood studies, CSF studies, nerve conduction studies, sural nerve biopsies, somatosensory evoked potentials (SEPs), and MRI of the cervical spinal cord including the C4 level on a $1.5 \mathrm{~T}$ unit. We used axial $\mathrm{T} 2{ }^{\star}$ weighted gradient echo images (repetition time/echo time/excitations, 700/21/3; flip angle, $20^{\circ}$; matrix, $256 \times 256$ ) as described previously. ${ }^{12} 1317$ We classified patients by MRI findings into three groups. In the first, MRI demonstrated a high intensity area in the posterior columns including both fasciculus cuneatus and gracilis. In the second, only the fasciculus gracilis showed high intensity. In the third, MRI showed no high intensity area in the posterior columns of the spinal cord.

Sural nerve biopsy specimens were examined by standard light microscopic methods and by teased fibre techniques. ${ }^{18}{ }^{19}$ Specimens fixed in $2.0 \%$ glutaraldehyde solution in 0.025 M cacodylate buffer ( $\mathrm{pH} 7.4$ ) or $10 \%$ buffered formalin solution were processed for histopathological assessment. Myelinated fibre density and morphological features were assessed in sections embedded in epoxy resin and stained with toluidine blue. Patients from whom specimens were processed for teased fibre study were assessed for pathological conditions according to criteria described previously. ${ }^{2021}$

\section{Results}

CLINICAL FINDINGS

Most of the patients (12 of 14) were diagnosed with Sjögren's syndrome after neurological symptoms appeared, having neuropathic symptoms before the onset of sicca symptoms. Only two patients were diagnosed with Sjögren's syndrome before neurological symptoms appeared. The initial symptom of neuronopathy was paraesthesias in the most distal portions of the limbs in 12 patients (feet three; hands nine). One limb was involved initially, and the other limbs became involved over a period of 4 months to 9 years. In two patients (patients 2 and 3), initial paraesthesias were localised to the trigeminal nerve distribution. Muscle strength remained essentially normal; patients $8,10,11$, and 14 had slight muscle weakness and atrophy. Muscle weakness found in the distal limbs could have been partly a consequence of involvement of joint and proprioceptive sensation, and muscle atrophy could be occurring from disuse.
In eight patients, deep sensation, (joint position sense and vibratory sense) was impaired more prominently than superficial sensation. These patients showed sensory ataxia in the limbs and trunk (table 1$).{ }^{22}$ Six patients showed similar degrees of superficial and deep sensory impairment.

The distribution of sensory impairment was essentially segmental, either in a spinal dermatome pattern or in a multiple mononeuritis pattern. In some patients, a glove and stocking pattern was seen, but an asymmetric distribution was apparent (table 1). Seven patients showed sensory impairment in the entire upper or lower limbs. In nine patients, sensory disturbance had expanded to the trunk. Deep tendon reflexes generally were diminished or absent.

As autonomic signs, five patients exhibited Adie's pupils, one anisocoria, and one elliptic pupils. Orthostatic hypotension was present in four patients. Segmental anhidrosis was seen in three patients, and in four patients loss of ${ }^{123}$ I-MIBG cardiac accumulation was seen (table 1).

\section{ELECTROPHYSIOLOGICAL AND PATHOLOGICAL} FINDINGS

Routine nerve conduction studies were performed in the median, tibial, and sural nerves in all patients. Motor nerve conduction studies in the median nerve and tibial nerve showed no slowing of conduction velocity in any patient, with respective ranges of 45 to $57 \mathrm{~m} / \mathrm{s}$ and 40 to $49 \mathrm{~m} / \mathrm{s}$. Amplitudes of compound muscle action potentials were reduced below $5 \mathrm{mV}$ in two patients in the median nerve and below 5 $\mathrm{mV}$ in one patient in the tibial nerve. $\mathrm{F}$ waves were normal in the median and tibial nerves. Sensory nerve action potentials (SNAPs) were not evoked in eight patients in the median nerve and in seven patients in the sural nerve. Slowing of sensory nerve conduction velocity was not seen in any nerve. These findings indicate that axonal degeneration and loss were prominent features in sensory nerves, whereas motor nerve conduction was substantially preserved.

Somatosensory evoked potentials (SEPs) were recorded in 11 patients using median nerve stimulation at the wrist. Cortical (N20), cervical (N13), and Erb's point (N9) peaks were not elicited in five, eight, and three patients respectively (table 1 ). N20 and N13 potentials were elicited less often than N9 potentials, indicating that the central pathway is severely involved in most patients.

Myelinated fibre density in the sural nerve was variably diminished (range 842-6663 fibres $/ \mathrm{mm}^{2}$ (mean (SD), $2808(1735) / \mathrm{mm}^{2}$; controls, $\left.8190(511) / \mathrm{mm}^{2}\right)$. Most patients predominantly lost large diameter myelinated fibres, (range for fibre number in patients 26 to $1159 / \mathrm{mm}^{2}$ (mean (SD), 406 (385) $/ \mathrm{mm}^{2}$; controls $\left.3068(294) / \mathrm{mm}^{2}\right)$. Small myelinated fibre density ranged from 553 to 5889 fibres $/ \mathrm{mm}^{2}$, (mean (SD), $2694(1610) / \mathrm{mm}^{2}$; controls, 5122 $\left.(438) / \mathrm{mm}^{2}\right)$. Axonal sprouting was either absent or very rare. In teased fibre preparations, the frequency of fibres with active axonal degeneration ranged from $0 \%$ to $32 \%$ (mean 


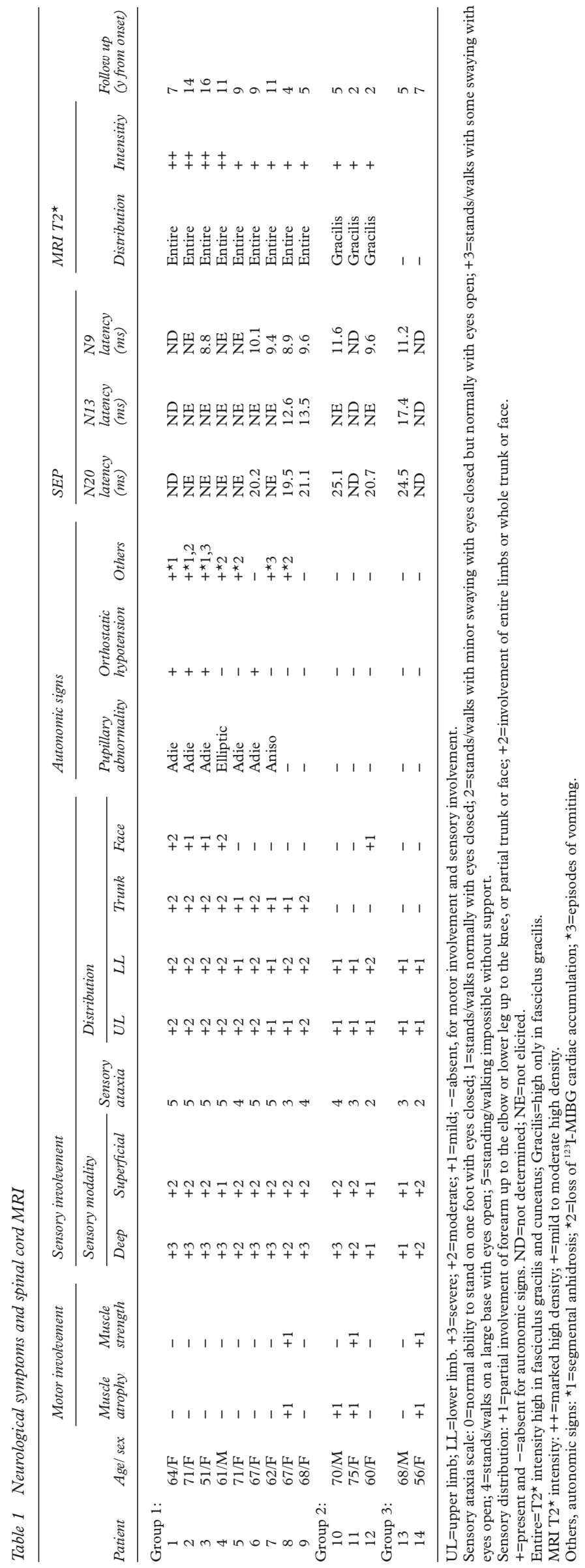

(SD), 12.4 (14.1)\%; controls, 1.7 (1.4)\%). Segmental demyelination was seen in 5\% to $28 \%$ of fibres (mean (SD), 11.4 (8.1)\%; controls, $9.5(8.8) \%)$. Chronic necrotising vasculitis was found in the epineurial space in two patients.

MRI FINDINGS AND THEIR CORRELATION WITH SENSORY AND AUTONOMIC SYMPTOMS

$\mathrm{T} 2 \star$ Images showed a high intensity area in the posterior column of the cervical cord in 12 patients. In nine patients (group 1), $\mathrm{T} 2{ }^{\star}$ weighted high intensity areas were seen in both the fasciculus cuneatus and gracilis (fig $1 \mathrm{~A}$ and B). In four of these nine patients the high intensity was marked; in the other five patients, intensity was moderately increased. In three of 12 patients, the high intensity area was restricted to the fasciculus gracilis (fig $1 \mathrm{C}$ and D; group 2). No MRI abnormalities were seen in two patients (group 3). The nine patients with high intensity areas in both fasciclus cuneatus and gracilis (group 1) showed an extensive distribution of the sensory deficit, involving entire upper and lower limbs and trunk as well as the face. Sensory ataxia was also profound. These patients also showed a high frequency of autonomic signs (table 1). Of these nine patients, seven showed a pupillary abnormality, five patients with Adie's pupils and two with elliptical pupils or anisocoria. Orthostatic hypotension was found in four of nine patients. Segmental anhidrosis, loss of ${ }^{123}$ I-MIBG cardiac accumulation, and episodes of vomiting were frequent in these nine patients (table 1). The interval between the first symptoms of neuronopathy and MRI ranged from 4 to 16 years (mean 9.6). On the other hand, the three patients with high intensity areas restricted to the fasciculus gracilis (group 2) showed sensory deficits distributed in the upper and lower limbs as a partial involvement, without involving the trunk (table 1). Signs of sensory ataxia were mild to moderate; autonomic dysfunction with pupillary abnormality, orthostatic hypotension, or other autonomic signs were not found in these three patients. The interval from neuronopathy onset was 2 to 5 years (mean 3.0). The two patients without high intensity areas in the posterior column (group 3) showed mild sensory disturbances restricted to portions of limbs, and did not show any autonomic signs (table 1). Duration of neuronopathy was 5 and 7 years.

When the ataxia scale and summated score of the sensory distribution were analysed in the three groups defined by MRI involvement, both ataxia scale and sensory distribution scores were significantly different between groups (fig 2). In group 1, with high intensity areas in both fasciculi, significantly more ataxia and wider sensory involvement was seen than in groups 2 and 3 with less or no MRI abnormality.

\section{Discussion}

We documented that high intensity areas in the spinal cord MRI in T2* weighted images were often present (12 of 14 patients) in the cervical dorsal columns of patients with neuronopathy 

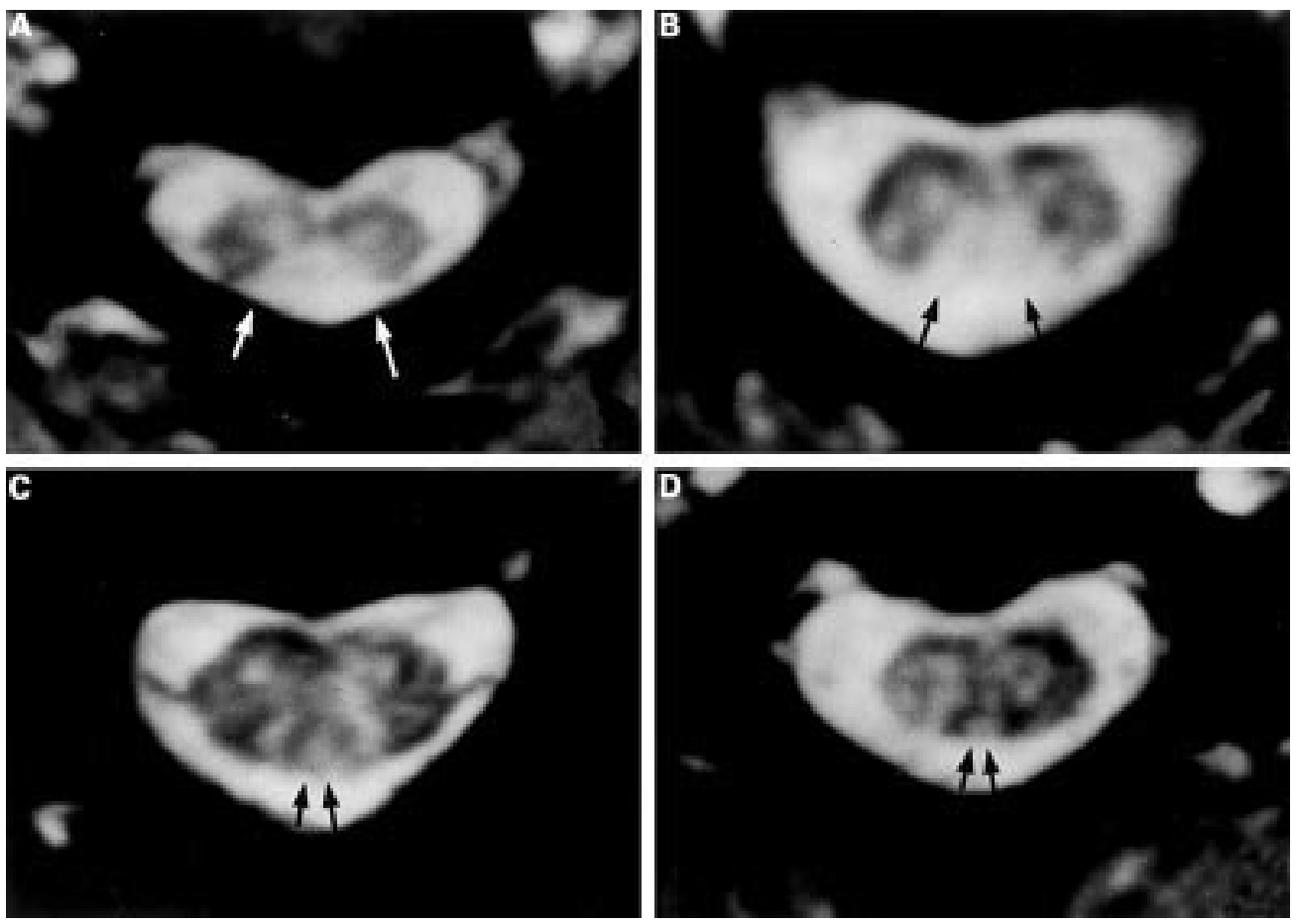

Figure 1 ( $A$ and $B)$ Axial T2* weighted gradient echo images of the cervical spinal cord (C4) of the patient. A high intensity area is present in the posterior column including both fasciculus cuneatus and gracilis as indicated by arrows. (C and D) Axial T2* weighted gradient echo images of the cervical spinal cord (C4) of the patient. A high intensity area is present in the posterior column including only fasciculus gracilis as indicated by arrows.

associated with Sjögren's syndrome. However, the extent and prominence of the high intensity area in the dorsal column was remarkably variable between patients, showing marked high intensity in both gracilis and cuneatus in four patients; mild high intensity in both gracilis and cuneatus in five patients; and high intensity only in the gracilis in three patients. In two patients, no high intensity MRI signals were seen in the dorsal columns. A striking finding was that the extent and intensity of high intensity areas in the dorsal columns correlated well with extent and distribution of sensory symptoms. Patients with high intensity areas in both gracilis and cuneatus (group 1) showed severe sensory disturbance with deep sensory involvement and sensory ataxia in the four limbs and trunk as well as distributed sensation in the face, whereas patients with high intensity areas

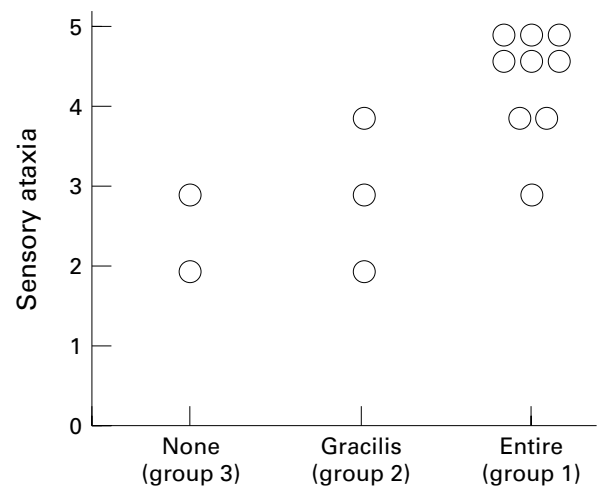

MRI T2 ${ }^{\star}$ findings only in the gracilis (group 2) showed sensory involvement in the limbs without involvement of the trunk. Furthermore, patients lacking high intensity areas in the dorsal columns (group 3) showed restricted sensory involvement in the limbs. Sensory evoked potentials, particularly $\mathrm{N} 20$ and N13, were not often evoked in patients with high intensity areas in both dorsal columns. These findings suggest that extent and intensity of spinal cord MRI findings represent extent of distribution of sensory involvement, particularly for deep sensory modalities. Because dorsal column involvement is suggested to be a secondary consequence of the dorsal root ganglionopathy, ${ }^{12} 1417$ the correlation between dorsal column MRI findings and sensory involvement also support the view that the major lesion responsible involves the dorsal root ganglion neurons. ${ }^{111} 13$

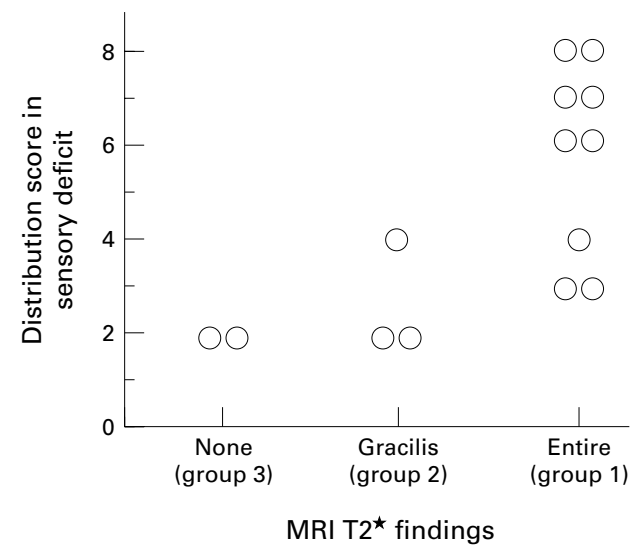

Figure 2 Functional status (ataxia scale) and sensory disturbance (summated score of the sensory distribution) among the three groups assessed with MRI. Patients in group 1 show high scores in both ataxia scale and distribution score of the sensory deficit $(p<0.05$ and $p<0.03$ respectively by Kruskal-Wallis test). 
Spinal dorsal column abnormalities with high intensity as demonstrated by $\mathrm{T} 2{ }^{\star} \mathrm{MRI}$, then, can be used to estimate the degree of sensory neuron involvement in neuronopathy associated with Sjögren's syndrome.

Anatomically, deep proprioceptive and kinaesthetic sensations are conducted through the dorsal columns; in humans, those from the lower limbs travel through the fasciculus gracilis and those from the upper limbs and trunk through the fasciculus cuneatus. ${ }^{23}$ Correlation of distribution of sensory symptoms, particularly those of deep sensation, with MRI documented distribution of the $\mathrm{T} 2{ }^{\star}$ high intensity areas are highly consistent with these anatomical patterns.

Another important finding was that the patients with longer follow up periods (more than 10 years) were often in group 1 , showing severe sensory deficits, and marked MRI hyperintensity. Furthermore, autonomic symptoms were present in group 1 but not in groups with less or no hyperintensity. These findings also suggest that MRI hyperintensity in the dorsal columns can serve as a marker for clinical severity in neuronopathy associated with Sjögren's syndrome.

Our present study also showed that the associated neuronopathy can be chronically progressive, over 16 years; such longstanding neuronopathy is accompanied by expansion of distribution of sensory symptoms, expansion of autonomic involvement, and expansion of dorsal column involvement evident by MRI. In the early stage, some patients respond well to corticosteroid therapy $^{11}$ or $\gamma$-globulin therapy. ${ }^{24}$ Thus, spinal cord MRI findings could also serve as markers for therapy. Absence of high intensity areas in the dorsal column might predict more likely benefit from intensive therapy, although further study is needed to clarify this point.

In summary, our findings have provided evidence that $\mathrm{T} 2^{\star}$ MRI high intensity abnormalities in the spinal dorsal columns represent severity of sensory neuronal involvement in neuronopathy associated with Sjögren's syndrome. These findings can estimate severity and may predict neurological outcome in this neuronopathy.

We are grateful to Dr T Yanagi (Department of Neurology, Nagoya Daini Red Cross Hospital, Nagoya); Dr S Terao (Fourth Department of Internal Medicine, Aichi Medical University, Aichi); Dr E Yoshida (Department of Neurology, Kasugai Municipal Hospital, Kasugai); and Dr S Mitake (Department of Neurology, Tosei General Hospital, Seto) for

1 Griffin JW, Cornblath DR, Alexander E, et al. Ataxic sensory neuropathy and dorsal root ganglionitis associated with neuropathy and dorsal root ganglionitis associa

2 Sobue G, Yasuda T, Kachi T, et al. Chronic progressive sensory ataxic neuropathy. Clinicopathological features of diopathic and Sjögren's syndrome-associated cases. $\mathcal{F} \mathrm{Neu}-$ rol 1993;240:1-7.

3 Grant IA, Hunder GG, Homburger HA, et al. Peripheral neuropathy associated with sicca complex. Neurology 1997; 48:855-62.

4 Kumazawa K, Sobue G, Yamamoto K, et al. Segmental anhidrosis in the spinal dermatomes in Sjögren's syndrome-associated neuropathy. Neurology 1993;43: 1820-3.

5 Attwood W, Poser CM. Neurologic complications of Sjögren's syndrome. Neurology 1961;11:1034-41.

6 Kaltreider HB, Talal N. The neuropathy of Sjögren's syndrome: trigeminal nerve involvement. Ann Intern Med 1969;70:751-62.

7 Alexander EL, Provost TT, Stevens MB, et al. Neurologic complications of primary Sjögren's syndrome. Medicine (Baltimore) 1982;61:247-57.

8 Peyronnard JM, Charron L, Beaudet F, et al. Vasculitic neuropathy in rheumatoid disease and Sjögren's syndrome. Neurology 1982;32:839-45.

9 Mellgren SI, Conn DL, Stevens JC, et al. Peripheral neuropathy in primary Sjögren's syndrome. Nerology 1989; 39:390-4.

10 Kaplan JG, Rosenberg R, Reinitz E, et al. Invited review: peripheral neuropathy in Sjögren's syndrome. Muscle Nerve 1990;13:570-9.

11 Malinow K, Yannakakis GD, Glusman SM, et al. Subacute sensory neuronopathy secondary to dorsal root ganglionitis in primary Sjögren's syndorome. Ann Neurol 1986;20:535-

12 Yasuda T, Sobue G, Hirose Y, et al. MR of acute autonomic and sensory neuropathy. Am $\mathcal{F}$ Neuroradiol 1994;15:114-5.

13 Sobue G, Yasuda T, Kumazawa K, et al. MRI demonstrates dorsal column involvement of the spinal cord in Sjögren's syndrome-associated neuropathy. Neurology 1995;45:5923 .

14 Lauria G, pareyson D, Grisoli $\mathrm{M}$, et al. Clinical and manetic resonance imaging findings in chronic sensory ganglionopathies. Ann Neurol 2000;47:104-9.

15 Research Committee of Sjögren's syndrome. Diagnostic criteria for Sjögren's syndrome. Annual report of Research Committee for Intractable Disease. Tokyo: Japanese Ministry of Health and Welfare, 1977:6.

16 Vitali C, Bombardieri S, Moutsopoulos HM, et al. Preliminary criteria for the classification of Sjögren's syndrome. Results of a prospective concerted action supported by the European community. Arthritis Rheum 1993;36:340-7.

17 Yasuda T, Sobue G, Hirose Y, et al. MRI findings in patients with acute autonomic and sensory neuropathy. Clin Neurol 1993;33:1075-8.

18 Sobue G, Hashizume Y, Mukai E, et al. X-linked recessive bulbospinal neuronopathy: a clinicopathological study. Brain 1989;112:209-32.

19 Hattori N, Ichimura M, Nagamatsu M, et al. Clinicopathological features of Churg-Strauss syndrome-associated neuropathy. Brain 1999;122:427-39.

20 Nagamatsu M, Terao S, Misu K, et al. Axonal and perikaryal involvement in chronic inflammatory demyelinating polyneuropathy. I Neurol Neurosurg Psychiatry 1999;66: $727-34$

21 Dyck PJ, Giannini C, Lais A. Pathologic alterations of nerves. In: Dyck PJ, Thomas PK, Griffin JW, et al, eds. Peripheral neuropathy. 3rd ed. Philadelphia: WB Saunders, 1993:514-95.

22 Nobile-Orazio E, Baldini L, Barbieri S, et al. Treatment of patients with neuropathy and anti-MAG IgM M-proteins. Ann Neurol 1988;24:93-7.

23 Brodal A. Neurological anatomy, in relation to clinical medicine, 3rd ed. New York: Oxford University Press, 1981:146-7.

24 Molina JA, Benito-Leon J, Bermejo F, et al. Intravenous immunoglobulin therapy in sensory neuropathy associated with Sjögren's the Psychiatry 1996;60:699. 